\title{
Association of ANK1 variants with new-onset type 2 diabetes in a Han Chinese population from northeast China
}

\author{
LULU SUN $^{1}$, XUELONG ZHANG $^{2}$, TONGTONG WANG ${ }^{1}$, MEIJUN CHEN $^{1}$ and HONG QIAO ${ }^{1}$ \\ ${ }^{1}$ Department of Endocrinology, The Second Affiliated Hospital; ${ }^{2}$ Laboratory of Medical Genetics, \\ Harbin Medical University, Harbin, Heilongjiang 150086, P.R. China
}

Received April 29, 2016; Accepted June 8, 2017

DOI: $10.3892 /$ etm.2017.4866

\begin{abstract}
Previous studies have identified three loci (rs4737009, rs515071 and rs516946) in ankyrin 1 (ANK1) that are associated with type 2 diabetes mellitus (T2DM) in a number of ethnic groups. However, the impact of single nucleotide polymorphisms (SNPs) of ANK1 on T2DM in a Han Chinese population from northeast China has not yet been studied. The present study was undertaken to investigate the relationship between the ANK1 gene and new-onset T2DM in northeastern China. Three widely studied variants were genotyped and analyzed for T2DM susceptibility in 1,962 Chinese subjects (996 with T2DM and 966 healthy controls). Genotyping was performed using SNPscan ${ }^{\mathrm{TM}}$. The single-locus analysis, identified differences in the expression of rs515071 and rs516946 between cases and controls, with an odds ratio (OR) of 1.31 [95\% confidence interval (CI), 1.10-1.55; $\mathrm{P}=0.002]$ and 1.32 (95\% CI, 1.09-1.61; $\mathrm{P}=0.005$ ) respectively, while there were no differences in the expression of rs4737009 between the groups. For the SNP of rs515071, the presence of AA or GA significantly reduced the risk of T2DM compared with GG (adjusted $\mathrm{P}=0.019, \mathrm{OR}=0.78 ; 95 \%$ CI, 0.63-0.96). With respect to rs516946, individuals carrying TT or CT exhibited a decreased risk of T2DM compared with those with the $\mathrm{CC}$ allele (adjusted $\mathrm{P}=0.040, \mathrm{OR}=0.79 ; 95 \% \mathrm{CI}$, 0.63-0.99). Furthermore, haplotype analysis indicated that the haplotype frequency of GC in T2DM cases was significantly higher than in controls $(\mathrm{P}=0.002, \mathrm{OR}=1.31 ; 95 \% \mathrm{CI}, 1.10-1.55)$. Furthermore, the rs516946-CC genotype was associated with a larger waist circumference $(\mathrm{P}=0.031)$. The present data indicated that ANK1 was a potential T2DM susceptibility gene in a Han Chinese population from northeastern China.
\end{abstract}

Correspondence to: Professor Hong Qiao, Department of Endocrinology, The Second Affiliated Hospital, Harbin Medical University, 246 Xuefu Road, Harbin, Heilongjiang 150086, P.R. China

E-mail: qiaoh0823@sina.com

Key words: type 2 diabetes mellitus, ankyrin 1, single nucleotide polymorphism, SNPscan, haplotype

\section{Introduction}

Type 2 diabetes mellitus (T2DM) is a complex metabolic disorder, in which patients experience hyperglycemia as a result of pancreatic $\beta$-cell dysfunction and insulin resistance (1). The number of patients with T2DM continues to increase globally (2). Although the exact etiology remains unknown, the complex interactions of multiple environmental/lifestyle risk factors and genetic variants are considered to be involved in the pathogenesis of the disease (3). Since 2007, the number of T2DM genes identified has increased markedly, due to the advent of genome-wide association studies (GWAS) (4). Altogether, GWAS have identified $>70$ genetic variants associated with T2DM (5). Parallel, high-powered genome-wide analyses of quantitative glycemic traits have provided alternative insights into the function of these loci, which are improving understanding of disease pathophysiology (6). The majority of these studies have been performed in European populations and although many of the identified gene variants have subsequently been replicated in other ethnicities (7), to the best of our knowledge, the overlap between T2DM genes in different ethnicities remains unknown.

Among the variants associated with glucose metabolism revealed by GWAS, ankyrin 1 (ANK1) was initially identified in a Japanese population in 2012 and was proposed to be associated with T2DM (8). ANK1 is located on the 8p11.1 human chromosome region, which is comprised of 55 introns and 42 exons (9). Ankyrins act as adaptors in linking the integral membrane proteins to the underlying spectrin-actin cytoskeleton and serve key functions in various activities including cell motility, activation and proliferation $(10,11)$. Previous studies have assessed the effect of ANK1 on susceptibility to T2DM and glycemic metabolism indicators in numerous populations $(8,12-14)$. Soranzo et al (12) performed a meta-analysis across 25 GWAS and 8 cohorts, demonstrated that rs 4737009 (located in intron 1) was associated with glycated hemoglobin (HbA1c) levels in people of European descent $\left(\mathrm{P}=6.11 \times 10^{-12}\right)$. Imamura et al (8) demonstrated that rs515071 (located in intron 40) was associated with T2DM in the Japanese population. Morris et al (15) reported that rs516946 (located in intron 41) was associated with T2DM in a large population of European descent. Subsequently, the analyses in Danish populations confirmed the association between the presence of SNP rs516946 and a future risk of T2DM (13). However, due to 
ethnic and/or environmental factors, the contribution of these variants to T2DM and glycemic traits remains controversial. Soranzo et al (12) conducted an analysis on non-diabetic adults of European descent and observed that common variants of the rs4737009 loci may impact HbAlc levels via erythrocyte biology, however no heightened risk of T2DM was detected $(\mathrm{P}=0.069 ; \mathrm{OR}=1.05 ; 95 \% \mathrm{CI}, 1.00-1.10)$. Furthermore, an imputation-based genome-wide association study indicated that the risk alleles of rs4737009 were not associated with T2DM or HbAlc levels in a Japanese population (8). Notably, a European study demonstrated the SNP rs4737009 may increase T2DM risk (15). To determine the reasons behind these discrepancies and improve understanding regarding the role of ANK1 in T2DM predisposition, it is important to understand the consequences of inheriting the variants in various ethnicities.

Previous studies primarily investigated individuals of European ancestry, thus little is known about the impact of these risk alleles on new-onset T2DM in northern Han Chinese populations. Based on the results of previous research, it was hypothesized that there may be an association between T2DM-associated SNPs in the northern Han Chinese population. Subsequently, a case-control study was performed and three SNPs (rs4737009, rs515071 and rs516946) for ANK1 were genotyped in a Han Chinese cohort from the northeast region.

\section{Patients and methods}

Study population. A total of 1,962 residents from the northeast region of China were enrolled in the cohort, including 996 patients with new-onset T2DM (61.4\% male; mean age, $46.11 \pm 12.55$ years) and 966 controls (58.2\% male; mean age, $42.94 \pm 11.68$ years). Patients with new-onset T2DM were recruited from the Department Of Endocrinology of the Second Affiliated Hospital of Harbin Medical University (Harbin, China). Controls with a fasting plasma glucose (FPG) concentration $<5.1 \mathrm{mmol} / 1$ and $\mathrm{HbA} 1 \mathrm{c}<6.0 \%$, who had no family history of T2DM, were enrolled from the Physical Examination Center of the Second Affiliated Hospital of Harbin Medical University or from outpatient clinics at the same hospital. All subjects in the study provided written informed consent and the study protocol was approved by the Ethics Committee of Harbin Medical University. T2DM was confirmed by reviewing medical records for symptoms and use of a fasting and/or glucose-tolerance test, according to the World Health Organization Diagnostic Criteria For Diabetes (16).

Exclusion criteria for cases were as follows: The presence of type 1 diabetes, gestational diabetes or any other type of diabetes; duration of T2DM $>1$ year; treatment of T2DM with insulin; acute diabetic complications or the presence of any other serious metabolic disease that might raise glucose levels. The exclusion criteria for the control group were as follows: The presence of heart disease; liver dysfunction; malignancy; other serious systemic disease; or the use of any drugs known to influence the metabolism of glucose and lipids.

Anthropometric and clinical measurements. Subjects were interviewed to determine family histories and medical histories. Standard physical examinations and laboratory assessments of T2DM risk factors were performed. Body mass index (BMI) was calculated as weight $(\mathrm{kg})$ divided by height ${ }^{2}\left(\mathrm{~m}^{2}\right)$. Waist-hip ratio (WHR) was calculated as waist circumference $(\mathrm{cm})$ divided by hip circumference $(\mathrm{cm})$. The homeostatic model assessment of insulin resistance (HOMA-IR) and HOMA of $\beta$-cell function (HOMA- $\beta$ ) were calculated following a method previously described by Matthews et al (17).

Peripheral venous blood samples were collected in tubes when all subjects were in a fasting state. Quantitative measurements of plasma insulin were performed using double-antibody radioimmunoassays (Insulin Radioimmunoassay kit; Cisbio, Codolet, France) (18). Fasting plasma glucose (FPG) was quantified using the glucose oxidase-peroxidase procedure as previous described (19). Serum total cholesterol (TC), triglyceride (TG), high-density lipoprotein (HDL) and low-density lipoprotein (LDL) levels were measured using an automatic biochemical analyzer (Modular DPP; Roche Diagnostics $\mathrm{GmbH}$, Basel, Switzerland). HbA1c levels were measured using high-performance liquid chromatography as previous described (20).

Genotyping. Genomic DNA was extracted from peripheral blood leukocytes using the TIANamp Genomic DNA kit (Tiangen Biotech Co., Ltd., Beijing, China). Three SNPs (rs4737009, rs515071 and rs516946) were genotyped, which had previously reported to be associated with T2DM in other populations $(8,12,21)$. SNPs were genotyped using an SNPscan $^{\text {TM }}$ kit (Genesky Biotech Co., Ltd., Shanghai, China). This kit was developed using patented SNP genotyping technology by Genesky Biotech Co., Ltd., and uses double ligation and multiplex fluorescence polymerase chain reaction. In order to validate the genotyping accuracy using SNPscan ${ }^{\mathrm{TM}}$, a $5 \%$ random sample of each group (cases and controls) were genotyped twice for all SNPs. In total, 100 pairs of blind duplicates were included and the concordance rate was $>98 \%$.

Statistical analysis. Data are presented as the mean \pm standard deviation or median (interquartile range), where appropriate. Statistical analyses were performed using SPSS 17.0 (SPSS, Inc., Chicago, IL, USA). Continuous clinical data were compared using the unpaired Student's t-test and a $\chi^{2}$ test was used to compare categorical variables. The allele frequencies between cases and controls were compared using a $\chi^{2}$ test, where appropriate. Genotype distributions between cases and controls were determined using logistic regression under additive, dominant and recessive models. Statistical evaluations for testing the genetic effects of association between case-control status and each individual SNP, as measured by OR and $95 \%$ CIs, were estimated using unconditional logistic regression following adjustment for age, sex and BMI. Linear regression was used to analyze associations between SNPs and clinical indicators. $\mathrm{P}<0.05$ was considered to indicate a statistically significant difference. The Hardy-Weinberg equilibrium test was performed as previously described (22). Linkage disequilibrium (LD) between the polymorphisms was quantified using Haploview version 4.2 (www.broad.mit.edu/mpg/haploview).

\section{Results}

Study population characteristics. The anthropometric and biochemical characteristics of the subjects are presented 
Table I. Clinical and laboratory characteristics of cases and controls.

\begin{tabular}{|c|c|c|c|}
\hline Characteristic & T2DM & Controls & P-value \\
\hline $\mathrm{n}$ & 996 & 966 & - \\
\hline Male, n (\%) & $612(61.4)$ & $562(58.2)$ & 0.140 \\
\hline Age, years & $46.11 \pm 12.55$ & $42.94 \pm 11.68$ & $<0.001$ \\
\hline Height, m & $1.68 \pm 0.08$ & $1.69 \pm 0.08$ & 0.080 \\
\hline $\mathrm{BMI}, \mathrm{kg} / \mathrm{m}^{2}$ & $25.8 \pm 3.58$ & $23.3 \pm 3.35$ & $<0.001$ \\
\hline Waist circumference, $\mathrm{mm}$ & $93.49 \pm 10.44$ & $81.26 \pm 10.89$ & $<0.001$ \\
\hline Hip circumference, $\mathrm{mm}$ & $99.48 \pm 7.44$ & $95.76 \pm 7.21$ & $<0.001$ \\
\hline WHR & $0.94 \pm 0.06$ & $0.85 \pm 0.07$ & $<0.001$ \\
\hline $\mathrm{SBP}, \mathrm{mmHg}$ & $130.17 \pm 17.50$ & $121.25 \pm 15.12$ & $<0.001$ \\
\hline $\mathrm{DBP}, \mathrm{mmHg}$ & $84.64 \pm 11.17$ & $79.23 \pm 9.67$ & $<0.001$ \\
\hline $\mathrm{FPG}, \mathrm{mmol} / \mathrm{l}$ & $10.03 \pm 3.41$ & $4.82 \pm 0.33$ & $<0.001$ \\
\hline $\mathrm{TC}, \mathrm{mmol} / \mathrm{l}$ & $5.00 \pm 1.29$ & $4.88 \pm 1.04$ & 0.030 \\
\hline $\mathrm{TG}, \mathrm{mmol} / \mathrm{l}$ & $2.38 \pm 2.25$ & $1.42 \pm 0.96$ & $<0.001$ \\
\hline HDL, mmol/1 & $1.21 \pm 0.32$ & $1.48 \pm 0.37$ & $<0.001$ \\
\hline $\mathrm{LDL}, \mathrm{mmol} / \mathrm{l}$ & $2.91 \pm 0.96$ & $2.92 \pm 0.86$ & 0.81 \\
\hline $\mathrm{FI}, \mu \mathrm{IU} / \mathrm{ml}$ & $12.89 \pm 7.59$ & $7.89 \pm 4.43$ & $<0.001$ \\
\hline $\mathrm{HbA} 1 \mathrm{c}, \%$ & $9.30 \pm 2.36$ & $5.12 \pm 0.47$ & $<0.001$ \\
\hline НОМА- $\beta$ & $38.86(22.09,69.85)$ & $104(70.33,149.12)$ & $<0.001$ \\
\hline HOMA-IR & $4.81(2.77,7.48)$ & $1.51(1.01,2.18)$ & $<0.001$ \\
\hline
\end{tabular}

Data are presented as mean \pm standard deviation or median (interquartile range). BMI, body mass index; WHR, waist-hip ratio; SBP, systolic blood pressure; DBP, diastolic blood pressure; FPG, fasting plasma glucose; TC, total cholesterol; TG, triglycerides; HDL, high-density lipoprotein cholesterol; LDL, low-density lipoprotein cholesterol; FI, fasting insulin; HbA1c, glycated hemoglobin; HOMA- $\beta$, homeostasis model assessment of $\beta$ cell function; HOMA-IR, HOMA of insulin resistance.

Table II. Association analysis of ankyrin 1 candidate single nucleotide polymorphisms for T2DM.

\begin{tabular}{|c|c|c|c|c|c|}
\hline Gene & Allele & T2DM, n (\%) & Control, n (\%) & P-value & OR $(95 \% \mathrm{CI})$ \\
\hline \multirow[t]{2}{*}{ rs4737009 } & $\mathrm{G}$ & 968 (48.6) & $934(48.3)$ & 0.875 & $0.99(0.87-1.11)$ \\
\hline & A & $1,024(51.4)$ & 998 (51.7) & & \\
\hline \multirow[t]{2}{*}{ rs515071 } & A & $290(14.6)$ & $352(18.2)$ & 0.002 & $1.31(1.10-1.55)$ \\
\hline & G & $1,702(85.4)$ & $1,580(81.8)$ & & \\
\hline \multirow[t]{2}{*}{ rs516946 } & $\mathrm{T}$ & $208(10.4)$ & $258(13.4)$ & 0.005 & $1.32(1.09-1.61)$ \\
\hline & $\mathrm{C}$ & $1,784(89.6)$ & $1,674(86.6)$ & & \\
\hline
\end{tabular}

CI, confidence interval; OR, odds ratio; T2DM, type 2 diabetes mellitus.

in Table I. The subjects, including 996 patients with T2DM and 966 non-diabetic controls, were genotyped for three typical ANK1 SNPs. Analyses were conducted to determine the association of these alleles with new-onset T2DM. There were 612 males and 384 females (age, $46.11 \pm 12.55$ years) in the case group and 562 males and 404 females (age, $42.94 \pm 11.68$ years) in the control group.

No significant differences were observed between the groups with respect to sex, height or LDL levels. However, the age, BMI and WHR of the cases were significantly higher than those of the controls $(\mathrm{P}<0.001)$. Cases had significantly higher systolic and diastolic blood pressure compared with controls $(\mathrm{P}<0.001)$, as well as a significantly increased waist and hip circumferences, FBG, fasting insulin, HbA1c, HOMA-IR, TC and TG levels $(\mathrm{P}<0.001)$. However, HDL levels and HOMA- $\beta$ were significantly lower in cases compared with controls $(\mathrm{P}<0.001)$.

Association of ANK1 variants with T2DM. The allele frequencies and genotype distributions of three polymorphisms in the ANK1 gene conformed to the Hardy-Weinberg equilibrium in cases and controls ( $\mathrm{P}>0.05)$. Statistical analysis indicated that rs515071 and rs516946 exhibited allelic differences between case and control groups, $(\mathrm{OR}=1.31$; 95\% CI, 1.10-1.55; $\mathrm{P}=0.002$ and $\mathrm{OR}=1.32 ; 95 \% \mathrm{CI}, 1.09-1.61 ; \mathrm{P}=0.005$, respectively; Table II). However, there were no allelic differences in rs4737009.

The effects of the genotypes of three SNPs were analyzed further under three different genetic models using logistic tests 
(Table III). It was observed that the SNPs rs515071 and rs516946 were associated with T2DM risk by the dominant model analysis. Furthermore, the association was still significant following adjustment for sex, age and BMI by logistic regression analysis $(\mathrm{P}<0.05)$. For the rs515071 SNP, patients with AA or GA had a significantly lower risk of T2DM compared with those with GG (adjusted $\mathrm{P}=0.019$, OR=0.78; 95\% CI, $0.63-0.96)$. With respect to rs516946, individuals carrying TT or CT had a significantly reduced risk of developing T2DM compared with those with $\mathrm{CC}$ (adjusted $\mathrm{P}=0.040, \mathrm{OR}=0.79$; 95\% CI, 0.63-0.99). However, no association was observed in any genetic models for the SNP rs437009 ( $>>0.05)$.

Analysis of haplotype associations. Haplotype analysis was performed to evaluate whether the combined effects of these SNPs influenced T2DM risk. Haplotypes were constructed on the basis of the genotype data from three SNPs using the Haploview software. Pairwise D values between SNPs and the reconstructed LD plots of the three SNPs are presented in Fig. 1. Two SNPs (rs515071 and rs516946) exhibited strong LD with each other and therefore formed a haplotype block (Fig. 1). Three haplotypes with a frequency of $>1 \%$ were identified (Table IV). Haplotype analysis identified that among the three haplotypes, the most common were GC and AT, the frequencies of which were $0.85 / 0.82\left(\chi^{2}=9.522, \mathrm{P}=0.002\right)$ and $0.10 / 0.13\left(\chi^{2}=8.174, P=0.0042\right)$, respectively. There were significant differences between the haplotypes in the T2DM cases and control subjects $(\mathrm{P}<0.05)$. The haplotype frequency of GC in T2DM cases was significantly higher compared with controls (OR=1.31; 95\% CI, 1.10-1.55), indicating that the haplotype GC was significantly associated with an enhanced risk of T2DM $(\mathrm{P}<0.05)$. No significant difference was observed in the distribution of the haplotypes AC and AT between the two groups $(\mathrm{P}>0.05$; Table IV).

Associations between SNPs and T2DM characteristics. The association between each genotype of the three SNPs in ANK1 and various clinical characteristics was analyzed. It was determined that the CC genotype of the rs516946 SNP was significantly associated with an increased waistline $(\mathrm{B}=0.819$, standard error $=0.380, \mathrm{P}=0.031$; Table $\mathrm{V})$. No associations were observed between rs4737009, rs515071 or rs516946 and any glycemic traits, including FPG, HOMA-IR or HOMA- $\beta$.

\section{Discussion}

Ankyrins were originally identified as critical structural components of the erythrocyte membrane in the late 1970s (23). A number of studies have investigated the role of ANK1 common variations in $\mathrm{T} 2 \mathrm{DM}(8,13,21)$.

Prior to the current study, SNP rs515071 was identified as a susceptibility variant for T2DM in the Japanese population (8). Subsequently, an analysis of European GWAS data identified an association between rs515071 and T2DM (8). In addition, Japanese researchers have observed that the risk allele for T2DM (rs515071-C) modestly reduced BMI in non-diabetic controls (8). In the present study, it was identified that ANK1 variants in rs515071 were associated with new-onset T2DM in the northern Han Chinese population, which is consistent with previous results. Therefore, rs515071 may be a common locus for 
Table IV. Distribution of haplotype frequencies between cases and controls.

\begin{tabular}{lccccc}
\hline Haplotype & T2DM frequency & Control frequency & $\chi^{2}$ & P-value & OR (95\% CI) \\
\hline GC & 0.85 & 0.82 & 9.522 & 0.002 & $1.31(1.10-1.55)$ \\
AT & 0.10 & 0.13 & 8.174 & 0.004 & $0.75(0.62-0.92)$ \\
AC & 0.04 & 0.05 & 1.097 & 0.290 & $0.85(0.63-1.15)$ \\
\hline
\end{tabular}

OR, odds ratio; CI, confidence intervals; T2DM, type 2 diabetes mellitus.

Table V. Association analysis between rs516946 genotypes and waist circumference.

\begin{tabular}{lccc}
\hline Genotype & $\begin{array}{c}\text { Waist } \\
\text { circumference, cm }\end{array}$ & B (SE) & P-value \\
\hline CC & $88.04 \pm 12.29$ & $0.819(0.380)$ & 0.031 \\
CT & $85.36 \pm 12.29$ & $-0.726(0.390)$ & 0.063 \\
TT & $84.65 \pm 12.41$ & $-1.491(1.284)$ & 0.246
\end{tabular}

Waist circumference data are presented as the mean \pm standard deviation. SE, standard error; B- and P-values were adjusted for age, sex and body mass index.

T2DM across multiethnic populations. However, in the current study, no association was observed between the SNP rs515071 and clinical parameters. The mechanisms by which SNPs in ANK1 contribute to T2DM remain unknown. ANK1 appears to serve a function in the organization of myofibrils during assembly and may cooperate with obscurin in mediating interactions between the sarcoplasmic reticulum and myofibrils. It is also expressed in some of the organs that are key for glucose metabolism (24). Furthermore, the membrane-binding domain of ankyrin1 associates with calcium-induced calcium-release channels of the endoplasmic/sarcoplasmic reticulum, including the inositol trisphosphate and ryanodine receptors (25-27). Thus, ANK1 may serve a function in the onset of T2DM by mediating metabolism and signal transduction. However, pathogenesis of the disease may differ between populations and further research is required to elucidate the role of the genetic variant rs515071 among different populations.

A number of studies have investigated the role of ANK1 rs516946 in T2DM with relatively consistent results. Morris et al (15) demonstrated that the SNP rs516946 was associated with T2DM in a large population of European descent. The risk allele $\mathrm{C}$ of rs516946 was confirmed in Europeans and was associated with reduced HOMA- $\beta$. Further analysis conducted in Danish males also identified that ANK1 rs516946 was associated with an increased risk of T2DM (13). These studies provide evidence for an association between ANK1 rs516946, and decreased insulinogenic and disposition indices. Likewise, a study conducted recently in Finland reported that the risk SNP rs516946 for T2DM was associated with fasting acetoacetate and $\beta$-hydroxybutyrate (14). It was hypothesized that rs516946 may be involved in energy metabolism and interfere with the utilization of glucose by increasing the decomposition of adipose, causing changes in plasma
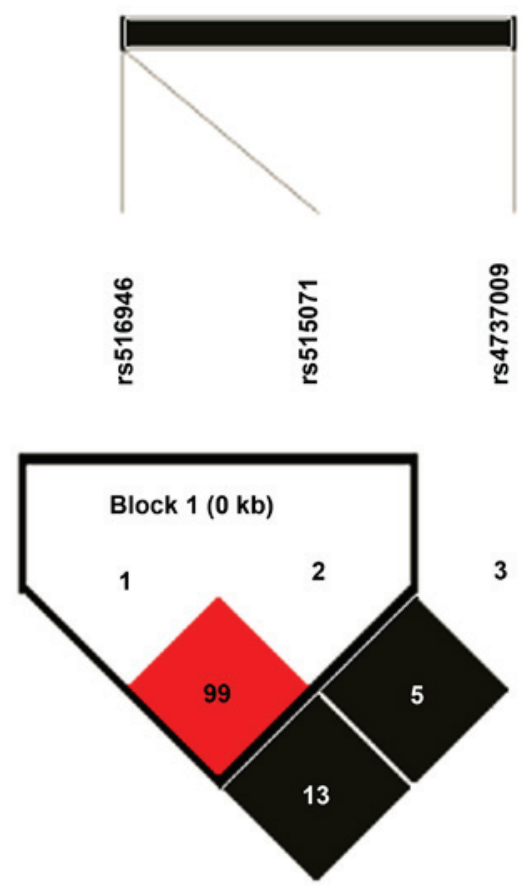

Figure 1. Linkage disequilibrium patterns of three genotyped single nucleotide polymorphisms of ankyrin 1 .

glucose. In the present study, it was also identified that genetic variant ANK1 (rs516946) was associated with the development of new-onset T2DM in a northeastern Han Chinese population. Furthermore, individuals with CC genotype had a larger waist circumference. As an important indicator in the diagnosis of obesity, waist circumference may be involved in the development of T2DM by influencing BMI $(28,29)$. This finding implies that ANK1 (rs516946) may increase T2DM susceptibility through its contribution to insulin resistance, which is experienced primarily by obese individuals. The specific pathological role of ANK1 in pancreatic $\beta$-cells has not yet been confirmed. However, the ANK1 protein links transmembrane proteins to the cytoskeleton and it has been suggested that rs516946 regulates ANK1 expression $(8,30)$.

It has been demonstrated that ANK1 rs4737009 lowers HbAlc levels by altering erythrocyte lifespan in non-diabetic European adults (12), however no association has been identified between SNP rs4737009 and T2DM. A meta-analysis across 12 GWAS in populations of European descent indicated that SNP rs4737009 may exert an influence on HbAlc and T2DM (30). However, Imamura et al (8) indicated that rs4737009 was not associated with T2DM or any glycometabolic indicators in a large Japanese sample. Consistent with 
the Japanese study, no association was observed between rs4737009 and T2DM or associated metabolic traits in the current study. Grimsby et al (31) reported that ethnic differences were observed in the association between ANK1 rs4737009 and HbA1c levels in adults from the USA. Thus, this variant may be ethnic-specific. Different results between East Asians and Europeans may be due to differences in environmental exposures, health care experiences and dietary structures between the two ethnic groups. Alternatively, gene-environment interactions may operate in the pathogenesis of T2DM and certain glucose metabolic traits. Thus more extensive analyses in multiethnic groups are required.

In the present study, haplotype analysis indicated that the frequencies of haplotype GC and AT differed significantly between cases and controls. The frequency of the haplotype GC was higher in the case group, suggesting it is a risk factor for T2DM. The results of the haplotype analysis were in agreement with the results of the comparison of genotypes and alleles of these SNPs (rs4737009, rs515071 and rs516946), suggesting that these gene variants are associated with new-onset T2DM in the northeastern Han Chinese population.

Potential limitations of the current study should be noted. As many participants as possible were recruited who met the study criteria of case and control, in order to avoid the reduction of study power. Additionally, matching procedures between the groups were not performed. As a result, there were significant differences in age and the sex ratio between cases and controls. Although these parameters were adjusting by including them in the same logistic model, these differences may have influenced the results of the association study. In addition, a small sample size suggested that further well-designed investigations are required to elucidate the role of the ANK1 SNPs in the development of T2DM.

In conclusion, the current study identified ANK1 as a significant locus associated with new-onset T2DM in a Han Chinese population from northeastern China. The ANK1 genotypes and haplotypes are associated with differing levels of risk for T2DM. Further studies and a larger sample size are required to elucidate the pathogenesis mechanism, as well as the potential contribution of other ANK1 variants to T2DM risk.

\section{Acknowledgements}

The authors gratefully acknowledge the valuable cooperation of Dr Yongjie Chen (Statistical Laboratory, Harbin Medical University, Harbin, China) during the use of data statistics. The current study was funded by the National Natural Science Foundation of China (grant nos. 81172742 and 81473053) and the Natural Science Foundation of Heilongjiang Province (grant no. ZD201220).

\section{References}

1. ADVANCE Collaborative Group; Patel A, MacMahon S, Chalmers J, Neal B, Billot L, Woodward M, Marre M, Cooper M, Glasziou $\mathrm{P}$, et al: Intensive blood glucose control and vascular outcomes in patients with type 2 diabetes. N Engl J Med 358: 2560-2572, 2008.

2. Guariguata L, Whiting DR, Hambleton I, Beagley J, Linnenkamp U and Shaw JE: Global estimates of diabetes prevalence for 2013 and projections for 2035. Diabetes Res Clin Pract 103: 137-149, 2014.
3. Franks PW: The complex interplay of genetic and lifestyle risk factors in type 2 diabetes: An overview. Scientifica (Cairo) 2012: 482186, 2012.

4. Wheeler E and Barroso I: Genome-wide association studies and type 2 diabetes. Brief Funct Genomics 10: 52-60, 2011.

5. Palmer ND, McDonough CW, Hicks PJ, Roh BH, Wing MR, An SS, Hester JM, Cooke JN, Bostrom MA, Rudock ME, et al: A genome-wide association search for type 2 diabetes genes in African Americans. PLoS One 7: e29202, 2012.

6. Dupuis J, Langenberg C, Prokopenko I, Saxena R, Soranzo N, Jackson AU, Wheeler E, Glazer NL, Bouatia-Naji N, Gloyn AL, et al: New genetic loci implicated in fasting glucose homeostasis and their impact on type 2 diabetes risk. Nat Genet 42: 105-116, 2010.

7. Mohlke KL and Boehnke M: Recent advances in understanding the genetic architecture of type 2 diabetes. Hum Mol Genet 24: R85-R92, 2015

8. Imamura M, Maeda S, Yamauchi T, Hara K, Yasuda K, Morizono T, Takahashi A, Horikoshi M, Nakamura M, Fujita H, et al: A single-nucleotide polymorphism in ANK1 is associated with susceptibility to type 2 diabetes in Japanese populations. Hum Mol Gen 21: 3042-3049, 2012.

9. Gallagher PG, Tse WT, Scarpa AL, Lux SE and Forget BG: Structure and organization of the human ankyrin-1 gene. Basis for complexity of pre-mRNA processing. J Biol Chem 272: 19220-19228, 1997.

10. Lambert $S$ and Bennett V: From anemia to cerebellar dysfunction. A review of the ankyrin gene family. Eur J Biochem 211: 1-6, 1993.

11. Bennett V and Baines AJ: Spectrin and ankyrin-based pathways: Metazoan inventions for integrating cells into tissues. Physiol Rev 81: 1353-1392, 2001.

12. Soranzo N, Sanna S, Wheeler E, Gieger C, Radke D, Dupuis J, Bouatia-Naji N, Langenberg C, Prokopenko I, Stolerman E, et al: Common variants at 10 genomic loci influence hemoglobin $A_{1}(C)$ levels via glycemic and nonglycemic pathways. Diabetes 59: 3229-3239, 2010.

13. Harder MN, Ribel-Madsen R, Justesen JM, Sparsø T, Andersson EA, Grarup N, Jørgensen T, Linneberg A, Hansen T and Pedersen O: Type 2 diabetes risk alleles near BCAR1 and in ANK1 associate with decreased beta-cell function whereas risk alleles near ANKRD55 and GRB14 associate with decreased insulin sensitivity in the Danish Inter99 cohort. J Clin Endocrinol Metab 98: E801-E806, 2013.

14. Abbasi A, Stolk RP and Bakker SJ: Identification of relevant biomarkers for type 2 diabetes. Lancet Diabetes Endocrinol 2: 106-107, 2014.

15. Morris AP, Voight BF, Teslovich TM, Ferreira T, Segrè AV, Steinthorsdottir V, Strawbridge RJ, Khan H, Grallert H, Mahajan A, et al: Large-scale association analysis provides insights into the genetic architecture and pathophysiology of type 2 diabetes. Nat Genet 44: 981-990, 2012

16. Medicine IOC: Definition and Diagnosis of Diabetes Mellitus and Intermediate Hyperglycemia. World Health Organization, Geneva, 2006.

17. Matthews DR, Hosker JP, Rudenski AS, Naylor BA, Treacher DF and Turner RC: Homeostasis model assessment: Insulin resistance and beta-cell function from fasting plasma glucose and insulin concentrations in man. Diabetologia 28: 412-419, 1985.

18. Silbert CK and Sawin CT: Double-antibody radioimmunoassay of serum insulin: Effect of use of hormone-depleted human serum. Clin Chem 21: 1520-1522, 1975.

19. Hill JB and Kessler G: An automated determination of glucose utilizing a glucose oxidase-peroxidase system. J Lab Clin Med 57: 970-980, 1961.

20. Davis JE, McDonald JM and Jarett L: A high-performance liquid chromatography method for hemoglobin A1c. Diabetes 27: 102-107, 1978.

21. Yan R, Lai S, Yang Y, Shi H, Cai Z, Sorrentino V, Du H and Chen H: A novel type 2 diabetes risk allele increases the promoter activity of the muscle-specific small ankyrin 1 gene. Sci Rep 6: 25105, 2016.

22. Nielsen DM, Ehm MG and Weir BS: Detecting marker-disease association by testing for Hardy-Weinberg disequilibrium at a marker locus. Am J Hum Genet 63: 1531-1540, 1998.

23. Bennett V: Purification of an active proteolytic fragment of the membrane attachment site for human erythrocyte spectrin. J Biol Chem 253: 2292-2299, 1978.

24. Busby B, Oashi T, Willis CD, Ackermann MA, KontrogianniKonstantopoulos A, Mackerell AD Jr and Bloch RJ: Electrostatic interactions mediate binding of obscurin to small ankyrin 1: Biochemical and molecular modeling studies. J Mol Biol 408: 321-334, 2011. 
25. Bourguignon LY, Chu A, Jin $\mathrm{H}$ and Brandt NR: Ryanodine receptor-ankyrin interaction regulates internal $\mathrm{Ca} 2^{+}$release in mouse T-lymphoma cells. J Biol Chem 270: 17917-17922, 1995.

26. Joseph SK and Samanta S: Detergent solubility of the inositol trisphosphate receptor in rat brain membranes. Evidence for association of the receptor with ankyrin. J Biol Chem 268: 6477-6486, 1993.

27. Mohler PJ, Davis JQ and Bennett V: Ankyrin-B coordinates the $\mathrm{Na} / \mathrm{K}$ ATPase, $\mathrm{Na} / \mathrm{Ca}$ exchanger, and InsP3 receptor in a cardiac T-tubule/SR microdomain. PLoS Biol 3: e423, 2005.

28. Misra A, Wasir JS and Vikram NK: Waist circumference criteria for the diagnosis of abdominal obesity are not applicable uniformly to all populations and ethnic groups. Nutrition 21: 969-976, 2005.
29. Feller S, Boeing $\mathrm{H}$ and Pischon T: Body mass index, waist circumference and the risk of type 2 diabetes mellitus: Implications for routine clinical practice. Dtsch Arztebl Int 107: 470-476, 2010.

30. Mehta NN: Large-scale association analysis provides insights into the genetic architecture and pathophysiology of type 2 diabetes mellitus. Circ Cardiovasc Genet 5: 708-710, 2012.

31. Grimsby JL, Porneala BC, Vassy JL, Yang Q, Florez JC, Dupuis J, Liu T, Yesupriya A, Chang MH, Ned RM, et al: Race-ethnic differences in the association of genetic loci with HbAlc levels and mortality in U.S. adults: The third National Health and Nutrition Examination Survey (NHANES III). BMC Med Genet 13: 30 , 2012. 